\title{
More on a topological mean value theorem
}

\author{
J.M. Almira, N. Del Toro, M. Jiménez \\ J.M. Almira obtained his Ph.D. at La Laguna University in 1999. Presently, he is \\ associate professor at the Department of Mathematics of Jaén University and co- \\ editor of La Gaceta de la R.S.M.E. His main interests are: approximation theory, \\ differential equations, new proofs, and the history of mathematics.
}

M. Jiménez graduated at Granada University in 1989. He also obtained a degree in management sciences at U.N.E.D. University in 2001. Presently, he works with J.M. Almira in his Ph.D. thesis. He is interested in mathematical modelling and optimization techniques.

N. Del Toro graduated at La Laguna University in 1999. Presently, she has a research grant from Junta de Andalucía to complete her Ph.D. thesis on approximation theory.

In her paper [3] I. Rosenholtz has proved, as a consequence of the Jordan curve theorem, the following interesting mean value theorem for the plane.

Theorem 1 (Rosenholtz) Let us assume that $\alpha:[a, b] \rightarrow \mathbb{R}^{2}$ is a differentiable non-stop arc in the plane (i.e., $\alpha$ is assumed to be injective and to have non zero derivative $\alpha^{\prime}(t)$ for all $t \in(a, b))$. Then there exists a positive number $M$ and $a t_{0} \in(a, b)$ such that $\alpha^{\prime}\left(t_{0}\right)=M(\alpha(b)-\alpha(a))$.

This theorem contains as a particular case the classical mean value theorem, which is usually stated for curves of the form $\alpha(t)=(t, f(t))$ and claims that if $f$ is continuous on $[a, b]$ and differentiable on $(a, b)$, there exists a point $t_{0} \in(a, b)$ such that the tangent

Der Mittelwertsatz der Differentialrechnung ist allen Lesern wohl vertraut. Ausgehend von einer Arbeit von I. Rosenholtz, die 1991 im American Mathematical Monthly erschien, beweisen die Autoren in dieser Arbeit zunächst, dass zu einer differenzierbaren Kurve $\alpha:[a, b] \rightarrow \mathbb{R}^{2}$ mit $\alpha$ injektiv und $\alpha^{\prime}(t) \neq 0$ für alle $t \in(a, b)$ eine Zahl $M \neq 0$ und ein $t_{0} \in(a, b)$ existieren, so dass $\alpha^{\prime}\left(t_{0}\right)=M(\alpha(b)-\alpha(a))$ gilt. Sie nennen dieses Ergebnis einen schwachen topologischen Mittelwertsatz. Ersetzt man dabei den $\mathbb{R}^{2}$ durch eine beliebige Fläche, so ist dieser Satz i.a. nicht mehr gültig, z.B. ist er für die 2-Sphäre verletzt. Als Hauptergebnis zeigen die Autoren nun, dass Flächen des $\mathbb{R}^{3}$, die mindestens einmal stetig differenzierbar sind und dem schwachen topologischen Mittelwertsatz genügen, genau die offenen Teilmengen von Ebenen des $\mathbb{R}^{3}$ sind. 
line to the curve $\alpha(t)=(t, f(t))$ at $\alpha\left(t_{0}\right)$ is parallel to the vector joining the end points of the curve, $(a, f(a))$ and $(b, f(b))$.

In her paper, Rosenholtz named Theorem 1 "Topological Mean Value Theorem" because its proof is strongly based on the Jordan curve theorem for the plane, and also because the result has some interesting topological consequences. For example, she proves that if $\alpha:[a, b] \rightarrow \mathbb{R}^{2}$ is a differentiable non-stop arc in the plane then the derivative directions: $\left\{\alpha^{\prime}(t) /\left\|\alpha^{\prime}(t)\right\|: t \in[a, b]\right\}$ form a connected subset of the unit circle (note that we are not assuming that $\alpha$ is of class $\mathbf{C}^{1}$ ) [3, Corol. 2]. In this note we respect this terminology.

On the other hand, it is very easy to prove a mean value type theorem which also holds for non Jordan curves. To be more precise, the following result holds:

Theorem 2 (Weak topological mean value theorem) Let us assume that $\alpha:[a, b] \rightarrow \mathbb{R}^{2}$ is a differentiable non-stop curve. Then there exists a number $M \neq 0$ and $a t_{0} \in(a, b)$ such that $\alpha^{\prime}\left(t_{0}\right)=M(\alpha(b)-\alpha(a))$.

Proof. There is no loss of generality if we assume that $[a, b]=[0, T], \alpha(0)=(0,0)$ and $\alpha(T)=(0, A)$. Then for each $t \in[0, T]$ the square of the distance from $\alpha(t)$ to the $y$-axis $\Lambda=\{(0, y): y \in \mathbb{R}\}$ is given by $\mathbf{d}(\alpha(t), \Lambda)^{2}=\alpha_{1}^{2}(t)$ and, since $[0, T]$ is compact, attains its maximum at some point $t_{0} \in[0, T]$. It follows that $\alpha_{1}^{\prime}\left(t_{0}\right)=0$ and hence, since $\alpha^{\prime}$ vanishes nowhere, the theorem follows.

Remark. If $\alpha(t)$ is of class at least $\mathbf{C}^{1}$, then there is another nice proof of Theorem 2. Indeed, we may proceed as follows: We assume that $[a, b]=[0, T], \alpha(0)=(0,0)$, $\alpha(T)=(0, A)$ and that $\alpha=\alpha(s)$ is parametrized by the arc-length parameter. It follows that $\alpha^{\prime}(s)=(\cos \theta(s), \sin \theta(s))$ for a certain continuous function $\theta:[0, T] \rightarrow \mathbb{R}$, and

$$
\alpha(s)=\left(\int_{0}^{s} \cos \theta(r) \mathrm{d} r, \int_{0}^{s} \sin \theta(r) \mathrm{d} r\right) \quad \text { for all } s
$$

(here we have used that $\alpha(0)=(0,0)$ ). Now, $\alpha(T)=(0, A)$ implies

$$
\int_{0}^{T} \cos \theta(r) \mathrm{d} r=0
$$

Hence, there exists some $s_{0} \in(0, T)$ such that $\cos \theta\left(s_{0}\right)=0$. This ends the proof.

We say that Theorem 2 is weaker than the topological mean value theorem since if we restrict ourselves to consider Jordan arcs, it says nothing about the sign of $M$.

As we have already said, in her proof of Theorem 1, Rosenholtz used the Jordan curve theorem. However it is interesting to notice that on the sphere, where the Jordan curve theorem holds, the analogous of Theorem 1 is false.

In this note we will prove that Theorem 1 is only possible for plane curves. In fact, we will prove that Theorem 2 characterizes the planes of $\mathbb{R}^{3}$ in a certain sense. To do this we first introduce the following concept: 
Definition $\mathbf{3}$ Let $\mathbf{S} \subset \mathbb{R}^{3}$ be a smooth surface of class at least $\mathbf{C}^{1}$. We say that $\mathbf{S}$ satisfies the weak topological mean value theorem if for each differentiable non-stop curve $\alpha:[a, b] \rightarrow \mathbf{S}$, there exists a number $M \neq 0$ and a $t_{0} \in(a, b)$ such that $\alpha^{\prime}\left(t_{0}\right)=$ $M(\alpha(b)-\alpha(a))$.

The main result in this note is the following:

Theorem 4 Let us assume that $\mathbf{S} \subset \mathbb{R}^{3}$ is a smooth surface of class at least $\mathbf{C}^{1}$ which satisfies the weak topological mean value theorem. Then $\mathbf{S}$ is an open subset of a plane.

Proof. Suppose that $\mathbf{S}$ is not an open subset of a plane. There is no loss of generality in assuming that $\mathbf{S}$ has a chart $\mathbf{x}: \mathbb{D}_{\delta}:=\left\{(u, v) \in \mathbb{R}^{2}: u^{2}+v^{2}<\delta\right\} \rightarrow \mathbf{S}$ of the form

$$
\mathbf{x}(u, v)=(u, v, f(u, v))
$$

for a certain function $f \in \mathbf{C}^{1}\left(\mathbb{D}_{\delta}\right)$, such that $f(0,0)=f_{u}(0,0)=f_{v}(0,0)=0$, and $f(a, b)=f_{0} \neq 0$ for a certain $(a, b) \in \mathbb{D}_{\delta}$. If the curve $\alpha(t)=\mathbf{x}(u(t), v(t))$ satisfies $\alpha(0)=(0,0,0), \alpha(T)=\left(a, b, f_{0}\right)$ and $\alpha^{\prime}\left(t_{0}\right)$ is parallel to $\left(a, b, f_{0}\right)$, then the curve $\beta(t)=(u(t), v(t))$ satisfies $\beta(0)=(0,0), \beta(T)=(a, b)$ and $\beta^{\prime}\left(t_{0}\right)$ is parallel to $(a, b)$. Furthermore, if $\beta^{\prime}\left(t_{0}\right)=\lambda(a, b)$ for a certain constant $\lambda \neq 0$, then the identity $\alpha^{\prime}\left(t_{0}\right)=\lambda\left(a, b, f_{0}\right)$ also holds. This obviously implies that

$$
f_{u}\left(\beta\left(t_{0}\right)\right) a+f_{v}\left(\beta\left(t_{0}\right)\right) b=f_{0} .
$$

Now, the function $h(u, v)=f_{u}(u, v) a+f_{v}(u, v) b$ is continuous on $\mathbb{D}_{\delta}$ and vanishes at $(u, v)=(0,0)$, so that there exists a point $\mathbf{p}_{0}=\left(u_{0}, v_{0}\right) \in \mathbb{D}_{\delta} \backslash\{(0,0)\}$ such that $h\left(\mathbf{p}_{0}\right) \neq f_{0}$. In fact, we can assume that

$$
\mathbf{p}_{0}=\varepsilon_{1}(a, b)+\varepsilon_{2}(b,-a)
$$

for certain constants $\varepsilon_{1}, \varepsilon_{2} \in(0,1)$. We set $\beta(t)=t(a, b)+\rho(t)(b,-a)$, where $\rho$ : $[0,1] \rightarrow[0,1]$ is a smooth function such that $\rho(0)=\rho(1)=0, \rho\left(\varepsilon_{1}\right)=\varepsilon_{2}$ and $t=\varepsilon_{1}$ is the unique critical point of $\rho$. Then

$$
\beta^{\prime}(t)=(a, b)+\rho^{\prime}(t)(b,-a)
$$

is parallel to $(a, b)$ only for $t=\varepsilon_{1}$. We define $\alpha(t)=\mathbf{x}(\beta(t))$.

It is clear that $\alpha$ is differentiable of class at least $\mathbf{C}^{1}$ and $\alpha^{\prime}(t) \neq(0,0,0)$ for all $t$. If $\alpha^{\prime}\left(t_{0}\right)$ is parallel to $\left(a, b, f_{0}\right)$, then $\beta^{\prime}\left(t_{0}\right)$ is parallel to $(a, b)$ and $f_{u}\left(\beta\left(t_{0}\right)\right) a+f_{v}\left(\beta\left(t_{0}\right)\right) b=f_{0}$. But $\beta^{\prime}\left(t_{0}\right)=\lambda(a, b)$ implies $t_{0}=\varepsilon_{1}$. Hence $\beta\left(t_{0}\right)=\mathbf{p}_{0}$ and

$$
f_{u}\left(\beta\left(t_{0}\right)\right) a+f_{v}\left(\beta\left(t_{0}\right)\right) b=h\left(\mathbf{p}_{0}\right) \neq f_{0},
$$

a contradiction.

Final Remark. The previous results do not need, in any way, more regularity than $\mathbf{C}^{1}$. In particular, no notion of curvature is necessary for the surface under consideration. In so far, Theorem 4 can also be regarded as a regularity result, since the conclusion is, in particular, that the validity of the weak topological mean value theorem implies smoothness of the surface. 
Acknowledgements. The proof of Theorem 2 was proposed by an anonymous referee. We are very grateful to him/her because of several useful comments.

\section{References}

[1] Boothby, W.M.: An introduction to differentiable manifolds and Riemannian geometry. Academic Press 1986.

[2] do Carmo, M.: Differential Geometry of Curves and Surfaces. Pearson Higher Education 1976.

[3] Rosenholtz, I.: A topological mean value theorem for the plane. Am. Math. Monthly 98 (1991) 2, $149-154$.

J.M. Almira, M. Jimenez and N. Del Toro

Departamento de Matemáticas

Universidad de Jaén

E.U.P. Linares

23700 Linares (Jaén), Spain

e-mail: jmalmira@ujaen.es 